Original Article

\title{
Long-Term Outcomes of Tricuspid Annuloplasty for Functional Tricuspid Regurgitation Associated with Degenerative Mitral Regurgitation: Suture Annuloplasty Versus Ring Annuloplasty Using a Flexible Band
}

\author{
Takashi Murashita, MD, Yukikatsu Okada, MD, PhD, Hideo Kanemitsu, MD, PhD, \\ Naoto Fukunaga, MD, Yasunobu Konishi, MD, Ken Nakamura, MD, \\ and Tadaaki Koyama, MD, PhD
}

\begin{abstract}
Purpose: We investigated the long-term outcomes of suture/ring tricuspid valve annuloplasty for functional tricuspid regurgitation associated with degenerative mitral regurgitation. Methods: We retrospectively reviewed patients who underwent flexible ring tricuspid valve annuloplasty $(n=120)$ or suture tricuspid valve annuloplasty $(n=42)$ for functional tricuspid regurgitation concomitant with surgery for degenerative mitral regurgitation (mean follow-up duration, $5.3 \pm 5.1$ years).

Results: The mean age of patients was $62.5 \pm 13.1$ years. Thirty-day mortality was zero in the suture group, and $0.8 \%$ in the ring group. Tricuspid regurgitation grade at discharge was lower in the ring group $(p=\mathbf{0 . 0 0 2})$. No difference was observed between survival and freedom from major cardiac/cerebrovascular adverse events between the groups. However, freedom from $\geq$ moderate tricuspid regurgitation was higher in the ring group (Log$\operatorname{rank} \boldsymbol{p}=\mathbf{0 . 0 0 3}$ ). From univariate analysis, the risk factors for $\geq$ moderate TR were suture annuloplasty and preoperative tricuspid regurgitation grade. No reoperation for recurrent tricuspid regurgitation occurred in either group because symptoms experienced by patients with recurrent tricuspid regurgitation were relatively insignificant.

Conclusion: Concomitant tricuspid annuloplasty using flexible bands offered improved durability than suture annuloplasty for preventing postoperative tricuspid regurgitation progression.
\end{abstract}

Keywords: tricuspid regurgitation, tricuspid annuloplasty, flexible band, suture annuloplasty

$\overline{\text { Department of Cardiovascular Surgery, Kobe City Medical Center }}$ General Hospital, Kobe, Hyogo, Japan

Received: October 20, 2013; Accepted: December 8, 2013

Corresponding author: Takashi Murashita, MD. Department of Cardiovascular Surgery, Kobe City Medical Center General Hospital, 2-1-1 Minatozimaminami, Chuo-ku, Kobe, Hyogo 650-0047, Japan

Email: tmurashita@gmail.com

(C)2014 The Editorial Committee of Annals of Thoracic and Cardiovascular Surgery. All rights reserved.

\section{Introduction}

Functional tricuspid regurgitation (TR) is often associated with left-sided valve disease, and it has historically been managed conservatively because of the expectation that it will diminish/disappear after surgical correction of left-sided valve pathology. ${ }^{1)}$ However, residual and recurrent functional TR has been reported after isolated mitral valve (MV) surgery, and severe late 
TR has been associated with an increase in early and midterm morbidity and mortality, in spite of adequate cardiac surgery for left-sided valve disease. ${ }^{2-6)}$

To control postoperative functional TR, concomitant tricuspid valve (TV) annuloplasty has been simultaneously attempted with MV surgery. However, few data regarding the long-term outcomes after TV annuloplasty are available, which has led to a variety of thresholds being used as an indication for its concomitant use. ${ }^{7,8)}$

Both suture annuloplasty and ring annuloplasty have been performed for treating TR, and a variety of prosthetic rings, such as flexible bands, semi-rigid rings, and rigid rings, are currently available. However, it is not clear which procedure is superior, and the durability of each prosthetic ring has not been described..$^{9-12)}$

In the early years, suture annuloplasty was usually used in TV annuloplasty procedure in our institution, but now the use of ring annuloplasty with flexible bands is predominant. Severe TR associated with degenerative MR was usually treated concomitantly with TV annuloplasty, whereas mild and moderate TR was treated on a caseto-case basis, according to the individual patient's needs. In cases of atrial fibrillation (AF)/pulmonary hypertension (PH), we have aggressively performed TV annuloplasty even for mild/moderate TR. In this study, we retrospectively investigated the long-term outcomes after TV annuloplasty for functional TR associated with degenerative MR.

\section{Materials and Methods}

The data analysis for this retrospective study was approved by the Institutional Review Board of Kobe City Medical Center General Hospital and the Board waived the need for patient consent.

\section{Patient population}

From January 1991 to December 2010, 1138 patients underwent MV surgery (949 MV repairs [MVRps] and 169 MV replacements [MVRs]) at Kobe City Medical Center General Hospital. Among patients who underwent MVRp, 247 (26.0\%) patients with type I/III MR were excluded. Patients with congenital MR and those who underwent concomitant aortic valve procedures were also excluded. As a result, 654 patients with type II degenerative MR remained in the study. Concomitant TV annuloplasty was performed on 162 patients $(24.8 \%)$. Of these, 42 underwent suture annuloplasty (suture group) and 120 underwent ring annuloplasty (ring group).

\section{Definitions}

Preoperative systolic pulmonary artery pressure (sPAP) was measured using Doppler echocardiography [modified Bernoulli equation: $4 \times(\text { TR jet velocity })^{2}+$ right atrial pressure $(10 \mathrm{mmHg})]$. $\mathrm{PH}$ was defined as $>50 \mathrm{mmHg}$ of sPAP. TR grade was scored as follows: none $/$ trivial $=0$, mild $=1$, moderate $=2$, and severe $=3$. Major cardiac and cerebrovascular adverse events are defined as: cardiac death, readmission for cardiac reasons, myocardial infarction, stroke, and transient ischemic attack.

\section{Clinical follow-up and examinations}

One hundred sixty (99.4\%) patients were followed up as outpatients or by telephone surveys. The mean followup duration was $9.1 \pm 4.2$ years in the suture group and $4.0 \pm 3.2$ years in the ring group. Postoperative transthoracic echocardiography was performed on an outpatient basis in 143 patients (89.4\%). Postoperative echocardiographic follow-up was generally performed before discharge and at $1,3,5,10$, and 15 years after operation. The mean duration of echocardiographic follow-up was $6.6 \pm 4.6$ years in the suture group and $2.9 \pm 3.2$ years in the ring group.

\section{Statistical analysis}

Continuous data in this study are expressed using mean \pm standard deviation and range. Categorical variables were compared with the $\chi^{2}$ or Fisher's exact tests, and continuous variables were compared with unpaired $t$ or Wilcoxon tests. Survival and freedom from major cardiac/cerebrovascular adverse events was calculated using the Kaplan-Meier method. A univariate Cox hazard regression analysis was used to identify predictors for late moderate TR. Statistical analysis was performed with StatView (SAS Institute, Cary, North Carolina, USA).

\section{Results}

\section{Preoperative characteristics (Table 1)}

The patients in the suture group were more symptomatic than those in the ring group: mean New York Heart Association (NYHA) functional class was $2.7 \pm 0.6$ in the suture group and $2.3 \pm 0.6$ in the ring group $(p<0.001)$. In addition, the suture group had a higher incidence of persistent AF.

Preoperative echocardiographic data showed that the suture group had a lower left ventricular (LV) ejection fraction and a higher left atrial (LA) diameter. There was 
Table 1 Patients' characteristics and preoperative echocardiographic data

\begin{tabular}{lccc}
\hline Variables & Suture $(\mathrm{n}=42)$ & Ring $(\mathrm{n}=120)$ & $p$-value \\
\hline Age $(\mathrm{y})$ & $64.0 \pm 10.4$ & $62.0 \pm 13.9$ & 0.389 \\
Male sex $(\mathrm{n}, \%)$ & $18(42.9 \%)$ & $76(63.3 \%)$ & 0.021 \\
Body surface area $\left(\mathrm{m}^{2}\right)$ & $1.54 \pm 0.20$ & $1.58 \pm 0.19$ & 0.294 \\
NYHA functional class (n, \%) & & \\
I & $1(2.4 \%)$ & $0(0 \%)$ & 0.582 \\
II & $12(28.6 \%)$ & $88(73.3 \%)$ & $<0.001$ \\
III & $27(64.3 \%)$ & $25(20.8 \%)$ & $<0.001$ \\
IV & $2(4.8 \%)$ & $7(5.8 \%)$ & 0.896 \\
Atrial fibrillation (n, \%) & $36(85.7 \%)$ & $67(55.8 \%)$ & $<0.001$ \\
LV diastolic diameter (mm) & $56.8 \pm 6.0$ & $55.7 \pm 7.5$ & 0.389 \\
LV systolic diameter (mm) & $36.6 \pm 6.5$ & $34.3 \pm 7.7$ & 0.088 \\
LV ejection fraction $(\%)$ & $62.6 \pm 9.5$ & $65.8 \pm 7.4$ & 0.040 \\
Left atrial diameter (mm) & $56.2 \pm 11.1$ & $51.8 \pm 10.6$ & 0.026 \\
Systolic PA pressure (mmHg) & $43.8 \pm 12.5$ & $46.9 \pm 17.3$ & 0.324 \\
Regurgitant volume at mitral valve (ml) & $60.0 \pm 63.7$ & $81.2 \pm 49.9$ & 0.058 \\
Regurgitant fraction at mitral valve $(\%)$ & $43.5 \pm 31.2$ & $55.9 \pm 24.9$ & 0.028 \\
Effective regurgitant orifice $\left(\mathrm{cm}^{2}\right)$ & $0.36 \pm 0.68$ & $0.59 \pm 0.71$ & 0.116 \\
Tricuspid regurgitation grade $(\mathrm{n}, \%)$ & & & \\
Mild & $17(40.5 \%)$ & $46(38.3 \%)$ & 0.806 \\
Moderate & $14(33.3 \%)$ & $40(33.3 \%)$ & 1.000 \\
Severe & $11(26.2 \%)$ & $34(28.3 \%)$ & 0.790 \\
\hline
\end{tabular}

NYHA: New York Heart Association; LV: left ventricle; PA: pulmonary artery

no statistical difference between the 2 groups with respect to $\mathrm{PH}$ or TR grade.

\section{Surgical techniques (Table 2)}

We used a median sternotomy in all patients. Standard cardiopulmonary bypass techniques, including bicaval cannulation, were then used. Myocardial protection was achieved using antegrade and retrograde intermittent cold-blood cardioplegia. Techniques used for MVRp are described by Carpentier ${ }^{13)}$ and David, et al. ${ }^{14)}$ Posterior leaflet prolapse was mostly corrected by resection and MV leaflet sutures. Anterior leaflet prolapse was mostly corrected using chordal replacement with polytetrafluoroethylene (Gore-Tex; W.L. Gore and Associates Inc., Flagstaff, Arizona, USA) sutures. MV ring annuloplasty was performed in the majority of patients.

All TV annuloplasty was performed under cardiac arrest. An oblique right atriotomy was placed to expose the TV. For suture annuloplasty, Kay sutures were performed in 30 patients and De Vega suture in 12 patients. In ring TV annuloplasty, interrupted 2-0 braided sutures without pledgets were placed circumferentially along the anterior, posterior, and right half of commissural leaflets. Flexible Duran annuloplasty ring (Medtronic Inc., Minneapolis, Minnesota, USA) was used in 87 patients; and flexible SJM Tailor annuloplasty ring was used (St. Jude
Medical, Inc., St. Paul, Minnesota, USA) in 33 patients. A saline infusion test was performed to confirm whether sufficient coaptation was obtained after ring annuloplasty. During the operation, transesophageal echocardiography was routinely performed, and the requirement for second pump run and intraoperative re-repair was judged on the basis of the findings. The mean ring size was $28.3 \mathrm{~mm} \pm 1.3 \mathrm{~mm}$ in Duran and $28.3 \mathrm{~mm} \pm 1.0 \mathrm{~mm}$ in Tailor $(p=0.794)$.

Concomitant procedures were performed in 91 patients (56.2\%). The most common procedure was the maze procedure (38.3\%), followed by LA appendage plication $(13.6 \%)$.

\section{Early outcomes}

No patient died within 30 days of follow-up in the suture group, but 1 patient in the ring group died (0.8\%) from LV rupture. In addition, 1 patient in the ring group died because of pneumonia during in-hospital stay.

In the suture group, the TR grade at discharge was trivial in 20 , mild in 17 , moderate in four, and severe in 1 patient. The mean TR grade was $0.8 \pm 0.7$ at discharge, which decreased from the preoperative value of $1.8 \pm$ $0.9(p<0.001)$. The TR grade in the ring group at discharge was trivial in 80 , mild in 36 and moderate in 2 patients; and the mean TR grade was $0.5 \pm 0.5$ at 
Table 2 Details of surgical procedure

\begin{tabular}{|c|c|c|c|}
\hline Variables & Suture $(n=42)$ & Ring $(n=120)$ & $p$-value \\
\hline Kay suture (n, \%) & $30(71.4 \%)$ & & \\
\hline De Vega suture $(n, \%)$ & $12(28.6 \%)$ & & \\
\hline Duran ring $(\mathrm{n}, \%)$ & & $87(72.5 \%)$ & \\
\hline $25 \mathrm{~mm}$ & & 4 & \\
\hline $27 \mathrm{~mm}$ & & 25 & \\
\hline $29 \mathrm{~mm}$ & & 53 & \\
\hline $31 \mathrm{~mm}$ & & 5 & \\
\hline Tailor ring (n, \%) & & $33(27.5 \%)$ & \\
\hline $27 \mathrm{~mm}$ & & 13 & \\
\hline $29 \mathrm{~mm}$ & & 20 & \\
\hline \multicolumn{4}{|l|}{ Concomitant other procedures } \\
\hline Maze procedure (n, \%) & $12(28.6 \%)$ & $50(41.7 \%)$ & 0.133 \\
\hline Left atrial appendage plication (n, \%) & $5(11.9 \%)$ & $17(14.2 \%)$ & 0.915 \\
\hline Closure of ASD or PFO (n, \%) & $4(9.5 \%)$ & $12(10.0 \%)$ & 0.833 \\
\hline Coronary artery bypass grafting $(\mathrm{n}, \%)$ & $2(4.8 \%)$ & $6(5.0 \%)$ & 0.725 \\
\hline Cardiopulmonary bypass time (minute) & $177.1 \pm 52.4$ & $180.0 \pm 50.2$ & 0.757 \\
\hline Aortic cross-clamp time (minute) & $121.0 \pm 35.6$ & $132.1 \pm 39.7$ & 0.111 \\
\hline
\end{tabular}

ASD: atrial septal defect; PFO: patent foramen ovale

discharge, which decreased from the preoperative value of $1.8 \pm 0.9(p<0.001)$. The TR grade at discharge was therefore lower in the ring group than in the suture group ( $p=0.002)$.

\section{Survival (Fig. 1)}

Survival did not differ between the 2 groups (Logrank $p=0.412$ ). In the suture group the 5-year and 10 -year survival were $90.4 \% \pm 4.6 \%$ and $79.9 \% \pm 7.0 \%$, respectively, and $93.5 \% \pm 3.4 \%$ and $84.8 \% \pm 6.8 \%$, respectively, in the ring group.

There were tenlate deaths in the suture group. Causes of death were cardiac failure in two, stroke in three, not cardiac related in four, and unknown in one. There were five late deaths in the ring group. The causes of death were stroke in three, arrhythmia in one, not cardiac related in one. All strokes that led to death occurred in patients with postoperative AF.

\section{Freedom from major cardiac/cerebrovascular adverse events}

Freedom from major cardiac/cerebrovascular adverse events was similar between the two groups (Log-rank $p=0.787$ ). Five-year and 10-year freedom was $87.6 \% \pm$ $5.2 \%$ and $70.1 \% \pm 8.2 \%$, respectively, in the suture group and $79.0 \% \pm 5.7 \%$ and $74.3 \% \pm 7.0 \%$, respectively, in the ring group.

Regarding hospital readmission, apart from the 5 patients who died because of cardiac causes in the suture group, 13 patients were readmitted for cardiac-related causes: five for congestive heart failure, four for implantation of a pacemaker for symptomatic bradycardia, three for a further cardiac reoperation related to the mitral/aortic valves, and one for myocardial infarction. In addition to the 15 patients who died because of cardiac causes in the ring group, 18 patients showed major cardiac/cerebrovascular adverse events during follow-up. Fourteen patients were readmitted for cardiac-related reasons: eight with congestive heart failure, four for implantation of a pacemaker in relation to symptomatic bradycardia, one for reoperation in relation to MV endocarditis, and one for catheter ablation in relation to postoperative AF. In addition to the three major strokes leading to death, 4 minor strokes occurred that did not result in death.

\section{Freedom from $\geq$ moderate $T R$ and risk factors for late TR}

Freedom from $\geq$ moderate TR (Fig. 2) was higher in the ring group than in the suture group (Log-rank $p=$ 0.003 ). Five-year and 10-year freedom was $61.6 \% \pm$ $8.5 \%$ and $40.9 \% \pm 10.5 \%$, respectively, in the suture group and $88.1 \% \pm 4.0 \%$ and $66.1 \% \pm 19.3 \%$, respectively, in the ring group. There was no reoperation for TV alone in both groups. There were five cases of progression to severe TR in the suture group, but in the ring group there were no cases of recurrence/progression to severe TR. 


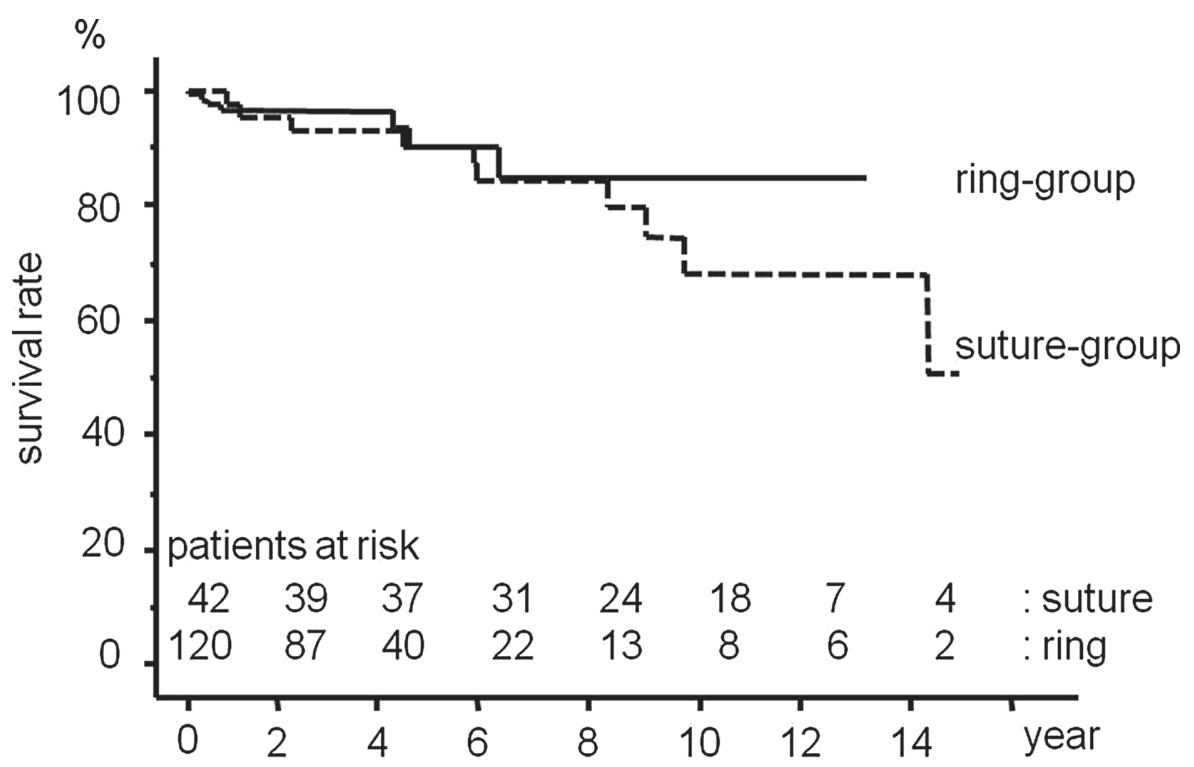

Fig. 1 Overall survival calculated by the Kaplan-Meier method. Solid line: ring group; dotted line: suture group.

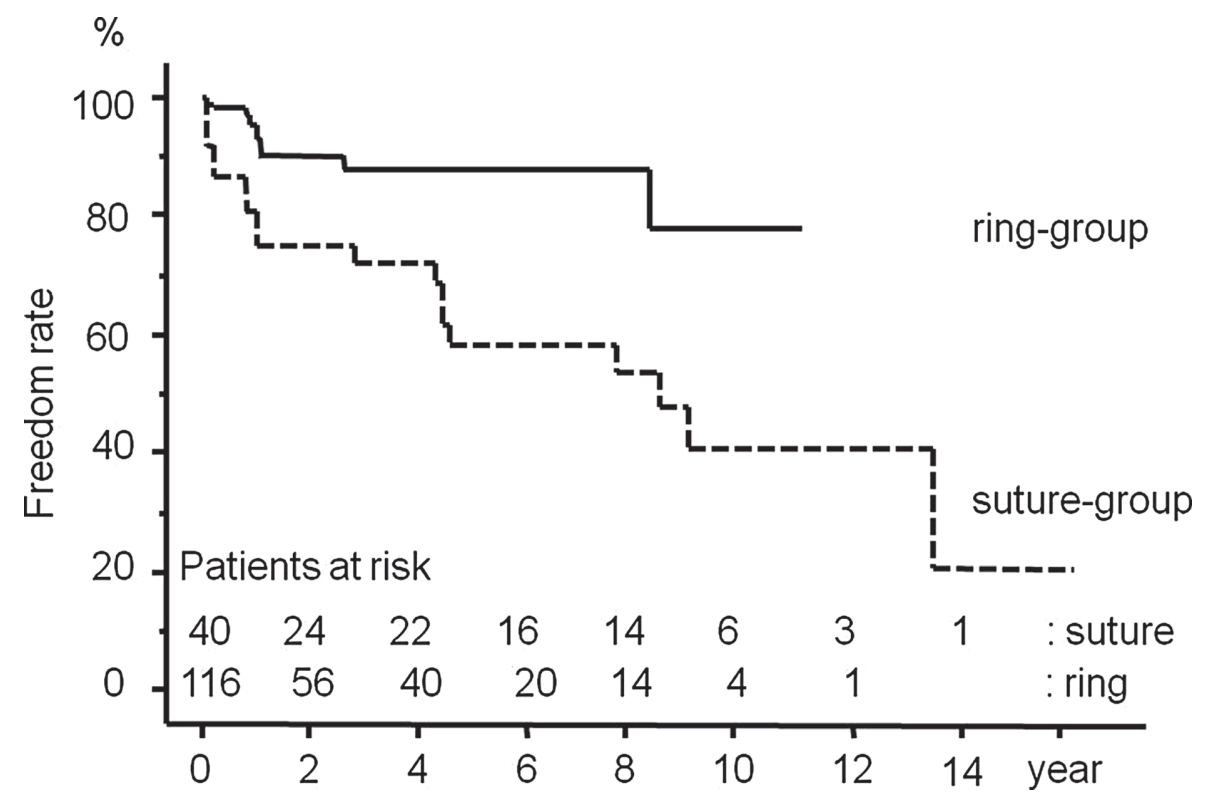

Fig. 2 Freedom from $\geq$ moderate tricuspid regurgitation (TR) calculated by Kaplan-Meier method. Solid line: ring group; dotted line: suture group.

The use of the procedures used in each group were compared in relation to freedom from $\geq$ moderate TR. Freedom was similar between Kay suture and the De Vega suture use (Log-rank $p=0.674)$ in the suture group (Fig. 3A), and between Duran ring and the Tailor ring use in the ring group (Log-rank $p=0.895$ ) (Fig. 3B).

However, univariate analysis did not clearly reveal that suture annuloplasty (Hazard ratio, 10.66; 95\% CI,
3.666-31.03; $p<0.001$ ) and preoperative TR grade (Hazard ratio, 2.816; 95\% CI, 1.399-5.663; $p=0.004)$ were risk factors for progression to moderate TR.

\section{Late symptoms and impact of late moderate TR}

In the suture group, the latest NYHA functional class for survivors was I in 27 patients $(87.1 \%)$ and II in 4 (12.9\%). The class in the 18 patients who progressed to $\geq$ moderate TR was I in 12 patients and II in 6 . Among 

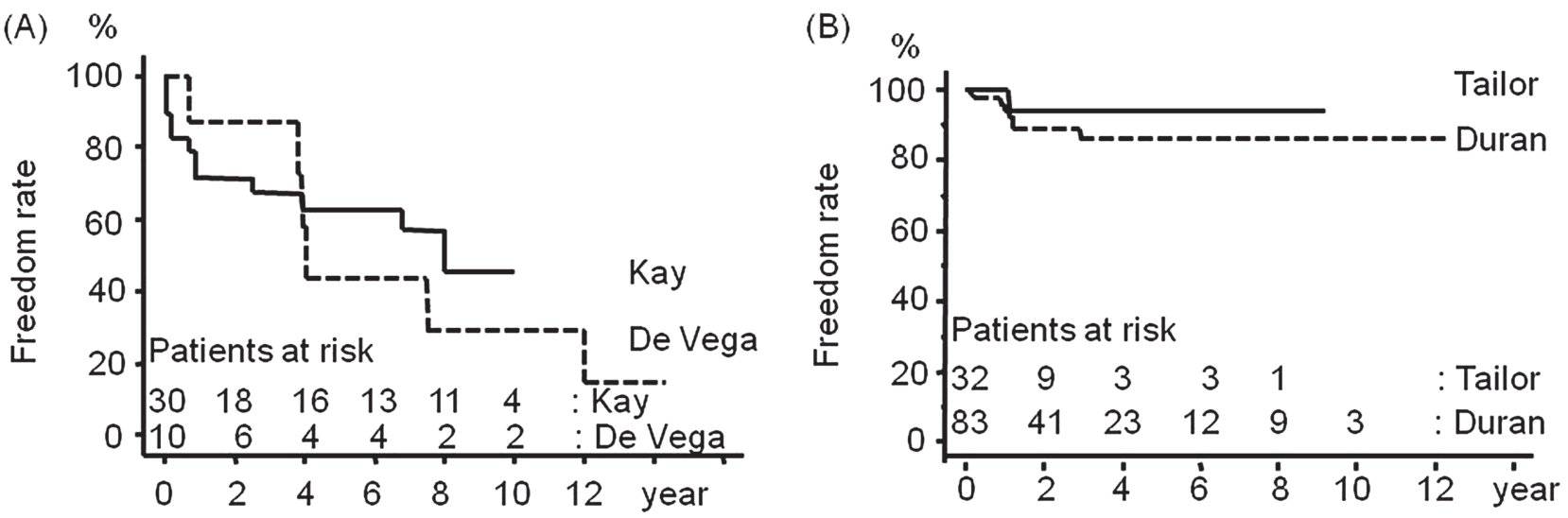

Fig. 3 Freedom from $\geq$ moderate tricuspid regurgitation (TR) calculated by Kaplan-Meier method in both groups. (A) Suture group. solid line: Kay suture; dotted line: De Vega suture. (B) Ring group. solid line: Tailor band; dotted line: Duran band.

the patients who progressed to $\geq$ moderate $T R$, no late deaths related to cardiac failure were observed. However, 10 patients required readmission for cardiac reasons. In the ring group, the latest NYHA functional class for survivors was I in 91 patients $(79.1 \%)$ and II in 24 $(20.9 \%)$. The class in the 10 patients who progressed to $\geq$ moderate TR was I in 6 patients and II in four. Among these 10 patients, three required readmission for congestive heart failure and one suffered a stroke.

\section{Discussion}

Functional TR is often associated with left-sided valve disease. Historically, functional TR has been managed conservatively. ${ }^{1,15)}$ However, moderate to severe late functional TR has been reported after isolated MV surgery. ${ }^{16,17)}$ Because severe late TR is associated with high mortality and morbidity, concomitant TV annuloplasty is recommended for severe TR at the time of MV surgery to prevent progression to functional TR. However, the use of concomitant TV annuloplasty for mild/moderate functional TR remains a controversial subject, ${ }^{7,8)}$ because limited available data related to the long-term outcomes of concomitant TV annuloplasty exist. Yilmaz, et al. concluded that TV annuloplasty is rarely necessary for degenerative MV disease because TR progression after MV surgery is unlikely. They insisted that progression of TR was clinically insignificant and did not lead to the risk of further surgery. ${ }^{7}$ However, Navia, et al. insisted that TV annuloplasty for moderate TR concomitant with mitral/aortic valve surgery was reasonable, because it provided an opportunity to prevent progression to and development of right ventricle dysfunction. ${ }^{8)}$
Since 1991, all patients with degenerative MR have undergone repair rather than replacement at our institution. We determine preoperatively whether TV annuloplasty is to be performed at the time of MVRp, in consideration of each patient's preoperative characteristics. Our initial attitude to TV annuloplasty was conservative. For mild/moderate TR, we mainly performed suture annuloplasty rather than ring annuloplasty. However, we encountered progression of TR after MVRp, although MR was well controlled. After these experiences, we began to employ a more aggressive approach for TR. Moreover, we have recently started performing TV annuloplasty for all moderate and severe functional TR using flexible bands. If a patient has $\mathrm{AF} / \mathrm{PH}$, we have also performed TV annuloplasty, even for mild TR. As a result, we have performed TV annuloplasty in approximately 25\% patients who underwent MVRp for degenerative MR. This number is much higher than that previously reported. ${ }^{8)}$

TV ring annuloplasty is mostly applicable to patients with functional TR. However, the incidences of residual and recurrent TR are reported to be high. McCarthy, et al. described 790 patients who underwent TV annuloplasty for functional TR and found that the incidence of residual TR was $15 \% 1$ month after repair. ${ }^{18)}$ Tang, et al. reported that there was a 30\% TR recurrence (among 702 patients) at a mean follow-up of 5.9 years after TV annuloplasty. ${ }^{19)}$

There were no differences in survival and freedom from major cardiac/cerebrovascular adverse events between the two groups. However, freedom from $\geq$ moderate TR was higher with ring annuloplasty than with suture annuloplasty. Even at discharge echocardiography, the TR 
grade was lower in the ring annuloplasty group, and there were no cases of progression to severe TR. Although some patients suffered from late moderate TR, the clinical symptoms were not significant. More than $50 \%$ patients who had moderate TR were in NYHA functional class I. Some patients required readmission for congestive heart failure, but the cause was mainly related to LV failure and not directly related to TR progression.

Many types of prosthetic rings are currently available: flexible bands, semi-rigid rings, and rigid rings. The choice of ring for use in TV annuloplasty for functional TR is controversial, and no randomized study regarding this has been conducted. Ghoreishi, et al. and Jeong, et al. reported successful outcomes for TV annuloplasty using undersized rigid 3-dimensional annuloplasty rings. ${ }^{9,10)}$ Benedetto, et al. reported on a randomized study hypothesizing whether TV annuloplasty should be added prophylactically at the time of MV surgery, and concluded that prophylactic TV annuloplasty using a flexible ring in patients with dilated tricuspid annulus was associated with a decreased incidence of TR progression. ${ }^{11)}$ Izutani, et al. reported on a retrospective study that compared the outcomes of using flexible bands and rigid rings ${ }^{12)}$ and concluded that although both flexible band and rigid ring annuloplasty provided a low incidence of TR recurrence, rigid ring annuloplasty may be more effective than flexible band annuloplasty for decreasing functional TR in immediate and mid-term postoperative periods.

However, the above studies offered only short- and mid-term outcomes. Although we have no experience of using rigid rings, in this study we have revealed satisfactory long-term outcomes for tricuspid annuloplasty using flexible bands.

Ring size selection is also a controversial topic. Ghoreishi, et al. reported that undersized rings optimally treated functional TR. ${ }^{9)}$ However, McCarthy, et al. found that tricuspid ring size did not predict late TR recurrence. ${ }^{18)}$

In our study, undersized TV annuloplasty was performed in most patients. Ring size selection was mainly determined by the patients' body surface area, not by the TV annular diameter. There were no clear criteria for ring selection. In general, 29-mm rings were used in patients with a large body surface area and $27-\mathrm{mm}$ rings were used in those with a small body surface area. However, on the basis of the positive long-term outcomes, we consider that our use of ring sizes has been effective.

It has been stated that tricuspid annulus dilatation is only possible in relation to its anterior and posterior aspects. ${ }^{16)}$ Although surgical procedures for TV ring implantation are varied, in previous reports TV annuloplasty has been performed with sutures only, along the anterior and posterior annulus. In our surgery, however, we always implant the annuloplasty ring by placing sutures along the anterior, posterior, and right half of the septal annulus. This implantation method has also been supported by a previous report ${ }^{9)}$ and is not a risk factor for pacemaker implantation. This implantation technique, in which sutures are also placed on the right half of the septal annulus, could be a reason why there has been less TR progression than in other reports. This technique may also be more efficient in preventing annular dilatation after the operation.

Several factors, such as female gender, ${ }^{3,7)}$ older age, $\left., 3,4\right)$ preoperative $\mathrm{AF},{ }^{7)}$ diabetes mellitus, ${ }^{7)}$ and severe preoperative $\mathrm{TR}^{20)}$ are reported to be associated with an increase in TR with time. Mutlak, et al. report that PH is a strong determinant of TR severity, but many patients with PH do not exhibit significant TR. ${ }^{21)}$ In this study, our univariate analysis showed that suture annuloplasty and preoperative TR grade were risk factors for late $\geq$ moderate $\mathrm{TR}$, whereas preoperative/postoperative AF and $\mathrm{PH}$ were not. This result shows the advantages of performing TV annuloplasty with flexible bands and how band use can overcome certain factors previously reported to cause deterioration in TR.

\section{Study limitations}

First, this study is a single-institution, retrospective review of collected data, predisposing to certain biases. Second, clinical and postoperative transthoracic echocardiography follow-up was not completed in all cases. Third, we did not intraoperatively measure the tricuspid annular diameter. Fourth, the right atrial and ventricular dimensions and degree of TV leaflet tethering were not studied because of lack of data. Finally, our technical ability of reproducibly quantifying TR could have been altered over the course of this study in line with developments in echocardiographic technology.

\section{Conclusion}

This study shows the long-term outcomes of TV annuloplasty for functional TR associated with degenerative MR. It is evident that concomitant TV ring annuloplasty using flexible bands offered improved durability than suture annuloplasty for preventing postoperative TR progression. 


\section{Acknowledgements}

The authors would like to thank Enago (www.enago.jp) for the English language review.

\section{Disclosure Statement}

The authors have no conflicts of interest to disclose.

\section{References}

1) Braunwald NS, Ross J, Morrow AG. Conservative management of tricuspid regurgitation in patients undergoing mitral valve replacement. Circulation 1967; 35: I63-9.

2) King RM, Schaff HV, Danielson GK, et al. Surgery for tricuspid regurgitation late after mitral valve replacement. Circulation 1984; 70: I193-7.

3) Porter A, Shapira Y, Wurzel M, et al. Tricuspid regurgitation late after mitral valve replacement: clinical and echocardiographic evaluation. J Heart Valve Dis 1999; 8: 57-62.

4) Staab ME, Nishimura RA, Dearani JA. Isolated tricuspid valve surgery for severe tricuspid regurgitation following prior left heart valve surgery: analysis of outcome in 34 patients. J Heart Valve Dis 1999; 8: 567-74.

5) Breyer RH, McClenathan JH, Michaelis LL, et al. Tricuspid regurgitation. A comparison of nonoperative management, tricuspid annuloplasty, and tricuspid valve replacement. J Thorac Cardiovasc Surg 1976; 72: 867-74.

6) Groves PH, Lewis NP, Ikram S, et al. Reduced exercise capacity in patients with tricuspid regurgitation after successful mitral valve replacement for rheumatic mitral valve disease. Br Heart J 1991; 66: 295-301.

7) Yilmaz O, Suri RM, Dearani JA, et al. Functional tricuspid regurgitation at the time of mitral valve repair for degenerative leaflet prolapse: the case for a selective approach. J Thorac Cardiovasc Surg 2011; 142: 608-13.

8) Navia JL, Brozzi NA, Klein AL, et al. Moderate tricuspid regurgitation with left-sided degenerative heart valve disease: to repair or not to repair? Ann Thorac Surg 2012; 93: 59-67; discussion 68-9.
9) Ghoreishi M, Brown JM, Stauffer CE, et al. Undersized tricuspid annuloplasty rings optimally treat functional tricuspid regurgitation. Ann Thorac Surg 2011; 92: 89-95; discussion 96.

10) Jeong DS, Kim KH. Tricuspid annuloplasty using the MC3 ring for functional tricuspid regurgitation. Circ J 2010; 74: 278-83.

11) Benedetto U, Melina G, Angeloni E, et al. Prophylactic tricuspid annuloplasty in patients with dilated tricuspid annulus undergoing mitral valve surgery. J Thorac Cardiovasc Surg 2012; 143: 632-8.

12) Izutani $H$, Nakamura $T$, Kawachi $K$. Flexible band versus rigid ring annuloplasty for functional tricuspid regurgitation. Heart Int 2010; 5: e13.

13) Carpentier A. Cardiac valve surgery - the "French correction". J Thorac Cardiovasc Surg 1983; 86: 323-37.

14) David TE, Bos J, Rakowski H. Mitral valve repair by replacement of chordae tendineae with polytetrafluoroethylene sutures. J Thorac Cardiovasc Surg 1991; 101: 495-501.

15) Duran CM, Pomar JL, Colman T, et al. Is tricuspid valve repair necessary? J Thorac Cardiovasc Surg 1980; 80: 849-60.

16) Dreyfus GD, Corbi PJ, Chan KM, et al. Secondary tricuspid regurgitation or dilatation: which should be the criteria for surgical repair? Ann Thorac Surg 2005; 79: $127-32$.

17) Izumi $C$, Miyake $M$, Takahashi $S$, et al. Progression of isolated tricuspid regurgitation late after left-sided valve surgery. Clinical features and mechanisms. Circ J 2011; 75: 2902-7.

18) McCarthy PM, Bhudia SK, Rajeswaran J, et al. Tricuspid valve repair: durability and risk factors for failure. J Thorac Cardiovasc Surg 2004; 127: 674-85.

19) Tang GH, David TE, Singh SK, et al. Tricuspid valve repair with an annuloplasty ring results in improved long-term outcomes. Circulation 2006; 114: I577-81.

20) Matsuyama K, Matsumoto M, Sugita T, et al. Predictors of residual tricuspid regurgitation after mitral valve surgery. Ann Thorac Surg 2003; 75: 1826-8.

21) Mutlak D, Aronson D, Lessick J, et al. Functional tricuspid regurgitation in patients with pulmonary hypertension: is pulmonary artery pressure the only determinant of regurgitation severity? Chest 2009; 135: 115-21. 